\title{
Search for the semileptonic decay $D^{0(+)} \rightarrow b_{1}(1235)^{-(0)} e^{+} \nu_{e}$
}

M. Ablikim, ${ }^{1}$ M. N. Achasov ${ }^{10, c}$ P. Adlarson, ${ }^{64}$ S. Ahmed, ${ }^{15}$ M. Albrecht, ${ }^{4}$ R. Aliberti ${ }^{28}$ A. Amoroso, ${ }^{63 a, 63 c}$ Q. An,${ }^{60,48}$ Anita, ${ }^{21}$ X. H. Bai,${ }^{54}$ Y. Bai, ${ }^{47}$ O. Bakina, ${ }^{29}$ R. Baldini Ferroli, ${ }^{23 a}$ I. Balossino, ${ }^{24 a}$ Y. Ban, ${ }^{38, k}$ K. Begzsuren, ${ }^{26}$ J. V. Bennett, ${ }^{5}$ N. Berger ${ }^{28}$ M. Bertani, ${ }^{23 a}$ D. Bettoni, ${ }^{24 a}$ F. Bianchi, ${ }^{63 a, 63 c}$ J. Biernat ${ }^{64} \mathrm{~J}$. Bloms,${ }^{57}$ A. Bortone, ${ }^{63 a, 63 c}$ I. Boyko, ${ }^{29}$ R. A. Briere ${ }^{5}$ H. Cai,${ }^{65}$ X. Cai,${ }^{1,48}$ A. Calcaterra, ${ }^{23 a}$ G. F. Cao, ${ }^{1,52}$ N. Cao, ${ }^{1,52}$ S. A. Cetin, ${ }^{51 b}$ J. F. Chang, ${ }^{1,48}$ W. L. Chang, ${ }^{1,52}$ G. Chelkov, ${ }^{29, b}$ D. Y. Chen, ${ }^{6}$ G. Chen, ${ }^{1}$ H. S. Chen, ${ }^{1,52}$ M. L. Chen, ${ }^{1,48}$ S. J. Chen, ${ }^{36}$ X. R. Chen, ${ }^{25}$ Y. B. Chen, ${ }^{1,48}$ Z. J. Chen, ${ }^{20,1}$ W. S. Cheng, ${ }^{63 c}$ G. Cibinetto, ${ }^{24 a}$ F. Cossio, ${ }^{63 c}$ X. F. Cui, ${ }^{37}$ H. L. Dai, ${ }^{1,48}$ J. P. Dai, ${ }^{42, g}$ X. C. Dai, ${ }^{1,52}$

A. Dbeyssi, ${ }^{15}$ R. B. de Boer, ${ }^{4}$ D. Dedovich, ${ }^{29}$ Z. Y. Deng, ${ }^{1}$ A. Denig, ${ }^{28}$ I. Denysenko, ${ }^{29}$ M. Destefanis,${ }^{63 a, 63 c}$

F. De Mori, ${ }^{63 a, 63 c}$ Y. Ding, ${ }^{34}$ C. Dong, ${ }^{37}$ J. Dong, ${ }^{1,48}$ L. Y. Dong, ${ }^{1,52}$ M. Y. Dong, ${ }^{1,48,52}$ S. X. Du, ${ }^{68}$ J. Fang, ${ }^{1,48}$ S. S. Fang, ${ }^{1,52}$ Y. Fang, ${ }_{1}^{1}$ R. Farinelli, ${ }^{24 a}$ L. Fava, ${ }^{63 b, 63 c}$ F. Feldbauer, ${ }^{4}$ G. Felici, ${ }^{23 a}$ C. Q. Feng, ${ }^{60,48}$ M. Fritsch, ${ }^{4}$ C. D. Fu, ${ }^{1}$ Y. Fu, ${ }^{1}$ X. L. Gao, ${ }^{60,48}$ Y. Gao ${ }^{61}$ Y. Gao ${ }^{38, k}$ Y. G. Gao, ${ }^{6}$ I. Garzia, ${ }^{24 a, 24 b}$ E. M. Gersabeck ${ }^{55}$ A. Gilman,${ }^{56}$ K. Goetzen, ${ }^{11}$ L. Gong, ${ }^{37}$ W. X. Gong, ${ }^{1,48}$ W. Gradl, ${ }^{28}$ M. Greco, ${ }^{63 a, 63 c}$ L. M. Gu, ${ }^{36}$ M. H. Gu, ${ }^{1,48}$ S. Gu, ${ }^{2}$ Y. T. Gu, ${ }^{13}$ C. Y. Guan, ${ }^{1,52}$ A. Q. Guo, ${ }^{22}$ L. B. Guo, ${ }^{35}$ R. P. Guo, ${ }^{40}$ Y. P. Guo, ${ }^{9, h}$ Y. P. Guo, ${ }^{28}$ A. Guskov, ${ }^{29}$ S. Han ${ }^{65}$ T. T. Han, ${ }^{41}$ T.Z. Han, ${ }^{9, h}$ X. Q. Hao, ${ }^{16}$

F. A. Harris, ${ }^{53}$ K. L. He ${ }^{1,52}$ F. H. Heinsius, ${ }^{4}$ C. H. Heinz ${ }^{28}$ T. Held, ${ }^{4}$ Y. K. Heng, ${ }^{1,48,52}$ M. Himmelreich, ${ }^{11, f}$ T. Holtmann, ${ }^{4}$ Y. R. Hou, ${ }^{52}$ Z. L. Hou, ${ }^{1}$ H. M. Hu, ${ }^{1,52}$ J. F. Hu ${ }^{42,8}$ T. Hu, ${ }^{1,48,52}$ Y. Hu, ${ }^{1}$ G. S. Huang, ${ }^{60,48}$ L. Q. Huang, ${ }^{61}$ X. T. Huang,${ }^{41}$ Y. P. Huang, ${ }^{1}$ Z. Huang, ${ }^{38, k}$ N. Huesken ${ }^{57}$ T. Hussain, ${ }^{62}$ W. Ikegami Andersson, ${ }^{64}$ W. Imoehl, ${ }^{22}$ M. Irshad, ${ }^{60,48}$ S. Jaeger, ${ }^{4}$ S. Janchiv, ${ }^{26, j}$ Q. Ji, ${ }^{1}$ Q. P. Ji, ${ }^{16}$ X. B. Ji, ${ }^{1,52}$ X. L. Ji ${ }^{1,48}$ H. B. Jiang, ${ }^{41}$ X. S. Jiang, ${ }^{1,48,52}$ X. Y. Jiang, ${ }^{37}$ J. B. Jiao, ${ }^{41}$ Z. Jiao, ${ }^{18}$ S. Jin, ${ }^{36}$ Y. Jin, ${ }^{54}$ T. Johansson, ${ }^{64}$ N. Kalantar-Nayestanaki, ${ }^{31}$ X. S. Kang,${ }^{34}$ R. Kappert, ${ }^{31}$ M. Kavatsyuk, ${ }^{31}$ B. C. Ke, ${ }^{43,1}$ I. K. Keshk, ${ }^{4}$ A. Khoukaz, ${ }^{57}$ P. Kiese, ${ }^{28}$ R. Kiuchi, ${ }^{1}$ R. Kliemt, ${ }^{11}$ L. Koch,${ }^{30}$ O. B. Kolcu, ${ }^{51 b, e}$ B. Kopf, ${ }^{4}$ M. Kuemmel, ${ }^{4}$ M. Kuessner, ${ }^{4}$ A. Kupsc ${ }^{64}$ M. G. Kurth, ${ }^{1,52}$ W. Kühn, ${ }^{30}$ J. J. Lane, ${ }^{55}$ J. S. Lange, ${ }^{30}$ P. Larin, ${ }^{15}$ L. Lavezzi, ${ }^{63 c}$ H. Leithoff, ${ }^{28}$ M. Lellmann, ${ }^{28}$ T. Lenz, ${ }^{28}$ C. Li ${ }^{39}$ C. H. Li ${ }^{33}$ Cheng Li,${ }^{60,48}$ D. M. Li ${ }^{68}$ F. Li, ${ }^{1,48}$ G. Li ${ }^{1}$ H. B. Li, ${ }^{1,52}$ H. J. Li, ${ }^{9}$ h J. L. Li, ${ }^{41}$ J. Q. Li, ${ }^{4} \mathrm{Ke} \mathrm{Li},{ }^{1}$ L. K. Li, ${ }^{1}$ Lei Li ${ }^{3}$ P.L. Li, ${ }^{60,48}$ P. R. Li, ${ }^{32}$ S. Y. Li ${ }^{50}$ W. D. Li, ${ }^{1,52}$ W. G. Li, ${ }^{1}$ X. H. Li ${ }^{60,48}$ X. L. Li ${ }^{41}$ Z. B. Li, ${ }^{49}$ Z. Y. Li ${ }^{49}$ H. Liang, ${ }^{6,48}$ H. Liang, ${ }^{1,52}$ Y. F. Liang, ${ }^{45}$ Y. T. Liang, ${ }^{25}$ L. Z. Liao, ${ }^{1,52}$ J. Libby, ${ }^{21}$ C. X. Lin, ${ }^{49}$ B. Liu, ${ }^{42, g}$ B. J. Liu, ${ }^{1}$ C. X. Liu, ${ }^{1}$ D. Liu ${ }^{60,48}$ D. Y. Liu, ${ }^{42, g}$ F. H. Liu, ${ }^{44}$ Fang Liu, ${ }^{1}$ Feng Liu, ${ }^{6}$ H. B. Liu, ${ }^{13}$ H. M. Liu, ${ }^{1,52}$ Huanhuan Liu, ${ }^{1}$ Huihui Liu, ${ }_{17}$ J. B. Liu, ${ }^{60,48}$ J. Y. Liu, ${ }^{1,52}$ K. Liu, ${ }^{1}$ K. Y. Liu ${ }^{34}$ Ke Liu, ${ }^{6}$ L. Liu, ${ }^{60,48}$ Q. Liu ${ }^{52}$ S. B. Liu, ${ }^{60,48}$ Shuai Liu, ${ }^{46}$ T. Liu ${ }^{1,52}$ X. Liu, ${ }^{32}$ Y. B. Liu, ${ }^{37}$ Z. A. Liu, ${ }^{1,48,52}$ Z. Q. Liu, ${ }^{41}$ Y. F. Long, ${ }^{38, k}$ X. C. Lou, ${ }^{1,48,52}$ F. X. Lu, ${ }^{16}$ H. J. Lu, ${ }^{18}$ J. D. Lu, ${ }^{1,52}$ J. G. Lu, ${ }^{1,48}$ X. L. Lu, ${ }^{1}$ Y. Lu, ${ }^{1}$ Y. P. Lu, ${ }^{1,48}$ C. L. Luo, ${ }^{35}$ M. X. Luo, ${ }^{67}$ P. W. Luo, ${ }^{49}$ T. Luo®, X. L. Luo, ${ }^{1,48}$ S. Lusso, ${ }^{63 \mathrm{c}}$ X. R. Lyu, ${ }^{52}$ F. C. Ma ${ }^{34}$ H. L. Ma $\odot,{ }^{1}$ L. L. Ma ${ }^{41}$ M. M. Ma, ${ }^{1,52}$ Q. M. Ma, ${ }^{1}$ R. Q. Ma, ${ }^{1,52}$ R. T. Ma ${ }^{52}$ X. N. Ma,${ }^{37}$ X. X. Ma ${ }^{1,52}$ X. Y. Ma ${ }^{1,48}$ Y. M. Ma ${ }^{41}$ F. E. Maas,${ }^{15}$ M. Maggiora,${ }^{63 a, 63 c}$ S. Maldaner,${ }^{28}$ S. Malde, ${ }^{58}$ Q. A. Malik, ${ }^{62}$ A. Mangoni, ${ }^{23 b}$ Y. J. Mao, ${ }^{38, k}$ Z. P. Mao, ${ }^{1}$ S. Marcello, ${ }^{63 a, 63 c}$ Z. X. Meng, ${ }^{54}$ J. G. Messchendorp, ${ }^{31}$

G. Mezzadri, ${ }^{24 a}$ T. J. Min ${ }^{36}$ R. E. Mitchell, ${ }^{22}$ X. H. Mo, ${ }^{1,48,52}$ Y. J. Mo, ${ }^{6}$ N. Yu. Muchnoi, ${ }^{10, c}$ H. Muramatsu, ${ }^{56}$

S. Nakhoul, ${ }^{11, \mathrm{f}}$ Y. Nefedov, ${ }^{29}$ F. Nerling, ${ }^{11, \mathrm{f}}$ I. B. Nikolaev, ${ }^{10, \mathrm{c}}$ Z. Ning, ${ }^{1,48}$ S. Nisar, ${ }^{8, \mathrm{i}}$ S. L. Olsen, ${ }^{52}$ Q. Ouyang, ${ }^{1,48,52}$ S. Pacetti, ${ }^{23 b, 23 c}$ X. Pan, ${ }^{9, h}$ Y. Pan,${ }^{55}$ A. Pathak, ${ }^{1}$ P. Patteri, ${ }^{23 a}$ M. Pelizaeus, ${ }^{4}$ H. P. Peng, ${ }^{60,48}$ K. Peters,,${ }^{11, \text { f }}$ J. Pettersson, ${ }^{64}$ J. L. Ping, ${ }^{35}$ R. G. Ping, ${ }^{1,52}$ A. Pitka, ${ }^{4}$ R. Poling, ${ }^{56}$ V. Prasad, ${ }^{60,48}$ H. Qi ${ }^{60,48}$ H. R. Qi ${ }^{50}$ M. Qi,${ }^{36}$ T. Y. Qi,${ }^{9}$ T. Y. Qi, ${ }^{2}$

S. Qian, ${ }^{1,48}$ W.-B. Qian, ${ }^{52}$ Z. Qian, ${ }^{49}$ C. F. Qiao, ${ }^{52}$ L. Q. Qin, ${ }^{12}$ X. S. Qin, ${ }^{4}$ Z. H. Qin, ${ }^{1,48}$ J. F. Qiu, ${ }^{1}$ S. Q. Qu, ${ }^{37}$

K. H. Rashid, ${ }^{62}$ K. Ravindran, ${ }^{21}$ C. F. Redmer, ${ }^{28}$ A. Rivetti, ${ }^{63 c}$ V. Rodin, ${ }^{31}$ M. Rolo, ${ }^{63 c}$ G. Rong, ${ }^{1,52}$ Ch. Rosner, ${ }^{15}$

M. Rump, ${ }^{57}$ A. Sarantsev, ${ }^{29, d}$ Y. Schelhaas, ${ }^{28}$ C. Schnier, ${ }^{4}$ K. Schoenning, ${ }^{64}$ M. Scodeggio, ${ }^{24 a}$ D. C. Shan,${ }^{46}$ W. Shan, ${ }^{19}$ X. Y. Shan,${ }^{60,48}$ M. Shao, ${ }^{60,48}$ C. P. Shen, ${ }^{9}$ P. X. Shen, ${ }^{37}$ X. Y. Shen, ${ }^{1,52}$ H. C. Shi, ${ }^{60,48}$ R. S. Shi, ${ }^{1,52}$ X. Shi, ${ }^{1,48}$ X. D. Shi, ${ }^{60,48}$ J. J. Song, ${ }^{41}$ Q. Q. Song, ${ }^{60,48}$ W. M. Song, ${ }^{27,1}$ Y.X. Song, ${ }^{38, k}$ S. Sosio, ${ }^{63 a, 63 c}$ S. Spataro, ${ }^{63 a, 63 c}$ F. F. Sui, ${ }^{41}$ G. X. Sun, ${ }^{1}$ J. F. Sun, ${ }^{16}$ L. Sun, ${ }^{65}$ S. S. Sun, ${ }^{1,52}$ T. Sun, ${ }^{1,52}$ W. Y. Sun, ${ }^{35}$ X. Sun, ${ }^{20,1}$ Y. J. Sun,${ }^{6,48}$ Y. K. Sun ${ }^{60,48}$ Y. Z. Sun, ${ }^{1}$ Z. T. Sun, ${ }^{1}$ Y. H. Tan ${ }^{65}$ Y. X. Tan, ${ }^{60,48}$ C. J. Tang, ${ }^{45}$ G. Y. Tang, ${ }^{1}$ J. Tang, ${ }^{49}$ V. Thoren, ${ }^{64}$ B. Tsednee, ${ }^{26}$ I. Uman, ${ }^{51 \mathrm{~d}}$ B. Wang, ${ }^{1}$ B. L. Wang, ${ }^{52}$ C. W. Wang, ${ }^{36}$ D. Y. Wang, ${ }^{38, k}$ H. P. Wang, ${ }^{1,52}$ K. Wang, ${ }^{1,48}$ L. L. Wang, ${ }^{1}$ M. Wang, ${ }^{41}$ M. Z. Wang, ${ }^{38, k}$ Meng Wang, ${ }^{1,52}$ W. H. Wang, ${ }^{65}$ W. P. Wang, ${ }^{60,48}$ X. Wang, ${ }^{38, k}$ X. F. Wang, ${ }^{32}$ X. L. Wang, ${ }^{9, \mathrm{~h}}$ Y. Wang, ${ }^{49}$ Y. Wang, ${ }^{60,48}$ Y. D. Wang, ${ }^{15}$ Y. F. Wang, ${ }^{1,48,52}$ Y. Q. Wang, ${ }^{1}$ Z. Wang, ${ }^{1,48}$ Z. Y. Wang, ${ }^{1}$ Ziyi Wang, ${ }^{52}$ Zongyuan Wang, ${ }^{1,52}$ D. H. Wei, ${ }^{12}$ P. Weidenkaff, ${ }^{28}$ F. Weidner, ${ }^{57}$ S. P. Wen, ${ }^{1}$ D. J. White, ${ }^{55}$ U. Wiedner, ${ }^{4}$ G. Wilkinson, ${ }^{58}$ M. Wolke, ${ }^{64}$ L. Wollenberg, ${ }^{4}$ J. F. Wu ${ }^{1,52}$ L. H. Wu, ${ }^{1}$ L. J. Wu, ${ }^{1,52}$ X. Wu $\odot,{ }^{9, h}$ Z. Wu, ${ }^{1,48}$ L. Xia, ${ }^{60,48}$ H. Xiao, ${ }^{9, h}$ S. Y. Xiao, ${ }^{1}$ Y. J. Xiao, ${ }^{1,52}$ Z. J. Xiao, ${ }^{35}$ X. H. Xie, ${ }^{38, k}$ Y. G. Xie, ${ }^{1,48}$ Y. H. Xie, ${ }^{6}$ T. Y. Xing, ${ }^{1,52}$ X. A. Xiong, ${ }^{1,52}$ G. F. Xu, ${ }^{1}$ J. J. Xu, ${ }^{36}$ Q. J. Xu, ${ }^{14}$ W. Xu, ${ }^{1,52}$ X. P. Xu, ${ }^{46}$

F. Yan, ${ }^{9, h}$ L. Yan, ${ }^{63 a, 63 c}$ L. Yan, ${ }^{9, h}$ W. B. Yan, ${ }^{60,48}$ W. C. Yan, ${ }^{68}$ Xu Yan, ${ }^{46}$ H. J. Yang, ${ }^{42, g}$ H. X. Yang, ${ }^{1}$ L. Yang, ${ }^{65}$

R. X. Yang, ${ }^{6,48}$ S. L. Yang, ${ }^{1,52}$ Y. H. Yang, ${ }^{36}$ Y. X. Yang, ${ }^{12}$ Yifan Yang, ${ }^{1,52}$ Zhi Yang, ${ }^{25}$ M. Ye, ${ }^{1,48}$ M. H. Ye, J. H. Yin, ${ }^{1}$ Z. Y. You, ${ }^{49}$ B.X. Yu, ${ }^{1,48,52}$ C. X. Yu, ${ }^{37}$ G. Yu, ${ }^{1,52}$ J. S. Yu, ${ }^{20,1}$ T. Yu, ${ }^{61}$ C.Z. Yuan, ${ }^{1,52}$ W. Yuan, ${ }^{63 a, 63 c}$ X. Q. Yuan, ${ }^{38, k}$ Y. Yuan, ${ }^{1}$ Z. Y. Yuan, ${ }^{49}$ C. X. Yue, ${ }^{33}$ A. Yuncu, ${ }^{51 b, a}$ A. A. Zafar, ${ }^{62}$ Y. Zeng, ${ }^{20,1}$ B. X. Zhang, ${ }^{1}$ Guangyi Zhang, ${ }^{16}$ H. H. Zhang, ${ }^{49}$ H. Y. Zhang, ${ }^{1,48}$ J. L. Zhang, ${ }^{66}$ J. Q. Zhang, ${ }^{4}$ J. W. Zhang, ${ }^{1,48,52}$ J. Y. Zhang, ${ }^{1}$ J. Z. Zhang, ${ }^{1,52}$ 
Jianyu Zhang,, ${ }^{1,52}$ Jiawei Zhang, ${ }^{1,52}$ L. Zhang, ${ }^{1}$ Lei Zhang, ${ }^{36}$ S. Zhang, ${ }^{49}$ S. F. Zhang, ${ }^{36}$ T. J. Zhang, ${ }^{42, g}$ X. Y. Zhang, ${ }^{41}$ Y. Zhang, ${ }^{58}$ Y. H. Zhang, ${ }^{1,48}$ Y. T. Zhang, ${ }^{60,48}$ Yan Zhang, ${ }^{60,48}$ Yao Zhang, ${ }^{1}$ Yi Zhang, ${ }^{9, \mathrm{~h}}$ Z. H. Zhang, ${ }^{6}$ Z. Y. Zhang, ${ }^{65}$ G. Zhao, ${ }^{1}$ J. Zhao, ${ }^{33}$ J. Y. Zhao, ${ }^{1,52}$ J. Z. Zhao, ${ }^{1,48}$ Lei Zhao, ${ }^{60,48}$ Ling Zhao, ${ }^{1}$ M. G. Zhao, ${ }^{37}$ Q. Zhao, ${ }^{1}$ S. J. Zhao, ${ }^{68}$ Y. B. Zhao, ${ }^{1,48}$ Y. X. Zhao, ${ }^{25}$ Z. G. Zhao, ${ }^{60,48}$ A. Zhemchugov, ${ }^{29, b}$ B. Zheng, ${ }^{61}$ J. P. Zheng, ${ }^{1,48}$ Y. Zheng, ${ }^{38, k}$ Y. H. Zheng, ${ }^{52}$ B. Zhong, ${ }^{35}$ C. Zhong, ${ }^{61}$ L. P. Zhou, ${ }^{1,52}$ Q. Zhou, ${ }^{1,52}$ X. Zhou, ${ }^{65}$ X. K. Zhou, ${ }^{52}$ X. R. Zhou, ${ }^{60,48}$ A. N. Zhu, ${ }^{1,52}$ J. Zhu, ${ }^{37}$ K. Zhu, ${ }^{1}$ K. J. Zhu, ${ }^{1,48,52}$ S. H. Zhu, ${ }^{59}$ W. J. Zhu, ${ }^{37}$ X. L. Zhu, ${ }^{50}$ Y. C. Zhu, ${ }^{60,48}$ Z. A. Zhu, ${ }^{1,52}$ B. S. Zou, ${ }^{1}$ and J. H. Zou ${ }^{1}$

\title{
(BESIII Collaboration)
}

\author{
${ }^{1}$ Institute of High Energy Physics, Beijing 100049, People's Republic of China \\ ${ }^{2}$ Beihang University, Beijing 100191, People's Republic of China \\ ${ }^{3}$ Beijing Institute of Petrochemical Technology, Beijing 102617, People's Republic of China \\ ${ }^{4}$ Bochum Ruhr-University, D-44780 Bochum, Germany \\ ${ }^{5}$ Carnegie Mellon University, Pittsburgh, Pennsylvania 15213, USA \\ ${ }^{6}$ Central China Normal University, Wuhan 430079, People's Republic of China \\ ${ }^{7}$ China Center of Advanced Science and Technology, Beijing 100190, People's Republic of China \\ ${ }^{8}$ COMSATS University Islamabad, Lahore Campus, Defence Road, \\ Off Raiwind Road, 54000 Lahore, Pakistan \\ ${ }^{9}$ Fudan University, Shanghai 200443, People's Republic of China \\ ${ }^{10}$ G.I. Budker Institute of Nuclear Physics SB RAS (BINP), Novosibirsk 630090, Russia \\ ${ }^{11}$ GSI Helmholtzcentre for Heavy Ion Research GmbH, D-64291 Darmstadt, Germany \\ ${ }^{12}$ Guangxi Normal University, Guilin 541004, People's Republic of China \\ ${ }^{13}$ Guangxi University, Nanning 530004, People's Republic of China \\ ${ }^{14}$ Hangzhou Normal University, Hangzhou 310036, People's Republic of China \\ ${ }^{15}$ Helmholtz Institute Mainz, Johann-Joachim-Becher-Weg 45, D-55099 Mainz, Germany \\ ${ }^{16}$ Henan Normal University, Xinxiang 453007, People's Republic of China \\ ${ }^{17}$ Henan University of Science and Technology, Luoyang 471003, People's Republic of China \\ ${ }^{18}$ Huangshan College, Huangshan 245000, People's Republic of China \\ ${ }^{19}$ Hunan Normal University, Changsha 410081, People's Republic of China \\ ${ }^{20}$ Hunan University, Changsha 410082, People's Republic of China \\ ${ }^{21}$ Indian Institute of Technology Madras, Chennai 600036, India \\ ${ }^{22}$ Indiana University, Bloomington, Indiana 47405, USA \\ ${ }^{23 a}$ INFN Laboratori Nazionali di Frascati, I-00044 Frascati, Italy \\ ${ }^{23 \mathrm{~b}}$ INFN Sezione di Perugia, I-06100 Perugia, Italy \\ ${ }^{23 \mathrm{c}}$ University of Perugia, I-06100 Perugia, Italy \\ ${ }^{24 a}$ INFN Sezione di Ferrara, I-44122 Ferrara, Italy \\ ${ }^{24 \mathrm{~b}}$ University of Ferrara, I-44122 Ferrara, Italy \\ ${ }^{25}$ Institute of Modern Physics, Lanzhou 730000, People's Republic of China \\ ${ }^{26}$ Institute of Physics and Technology, Peace Ave. 54B, Ulaanbaatar 13330, Mongolia \\ ${ }^{27}$ Jilin University, Changchun 130012, People's Republic of China \\ ${ }^{28}$ Johannes Gutenberg University of Mainz, Johann-Joachim-Becher-Weg 45, D-55099 Mainz, Germany \\ ${ }^{29}$ Joint Institute for Nuclear Research, 141980 Dubna, Moscow region, Russia \\ ${ }^{30}$ Justus-Liebig-Universitaet Giessen, II. Physikalisches Institut, \\ Heinrich-Buff-Ring 16, D-35392 Giessen, Germany \\ ${ }^{31}$ KVI-CART, University of Groningen, NL-9747 AA Groningen, The Netherlands \\ ${ }^{32}$ Lanzhou University, Lanzhou 730000, People's Republic of China \\ ${ }^{33}$ Liaoning Normal University, Dalian 116029, People's Republic of China \\ ${ }^{34}$ Liaoning University, Shenyang 110036, People's Republic of China \\ ${ }^{35}$ Nanjing Normal University, Nanjing 210023, People's Republic of China \\ ${ }^{36}$ Nanjing University, Nanjing 210093, People's Republic of China \\ ${ }^{37}$ Nankai University, Tianjin 300071, People's Republic of China \\ ${ }^{38}$ Peking University, Beijing 100871, People's Republic of China \\ ${ }^{39}$ Qufu Normal University, Qufu 273165, People's Republic of China \\ ${ }^{40}$ Shandong Normal University, Jinan 250014, People's Republic of China \\ ${ }^{41}$ Shandong University, Jinan 250100, People's Republic of China \\ ${ }^{42}$ Shanghai Jiao Tong University, Shanghai 200240, People's Republic of China \\ ${ }^{43}$ Shanxi Normal University, Linfen 041004, People's Republic of China \\ ${ }^{44}$ Shanxi University, Taiyuan 030006, People's Republic of China \\ ${ }^{45}$ Sichuan University, Chengdu 610064, People's Republic of China
}




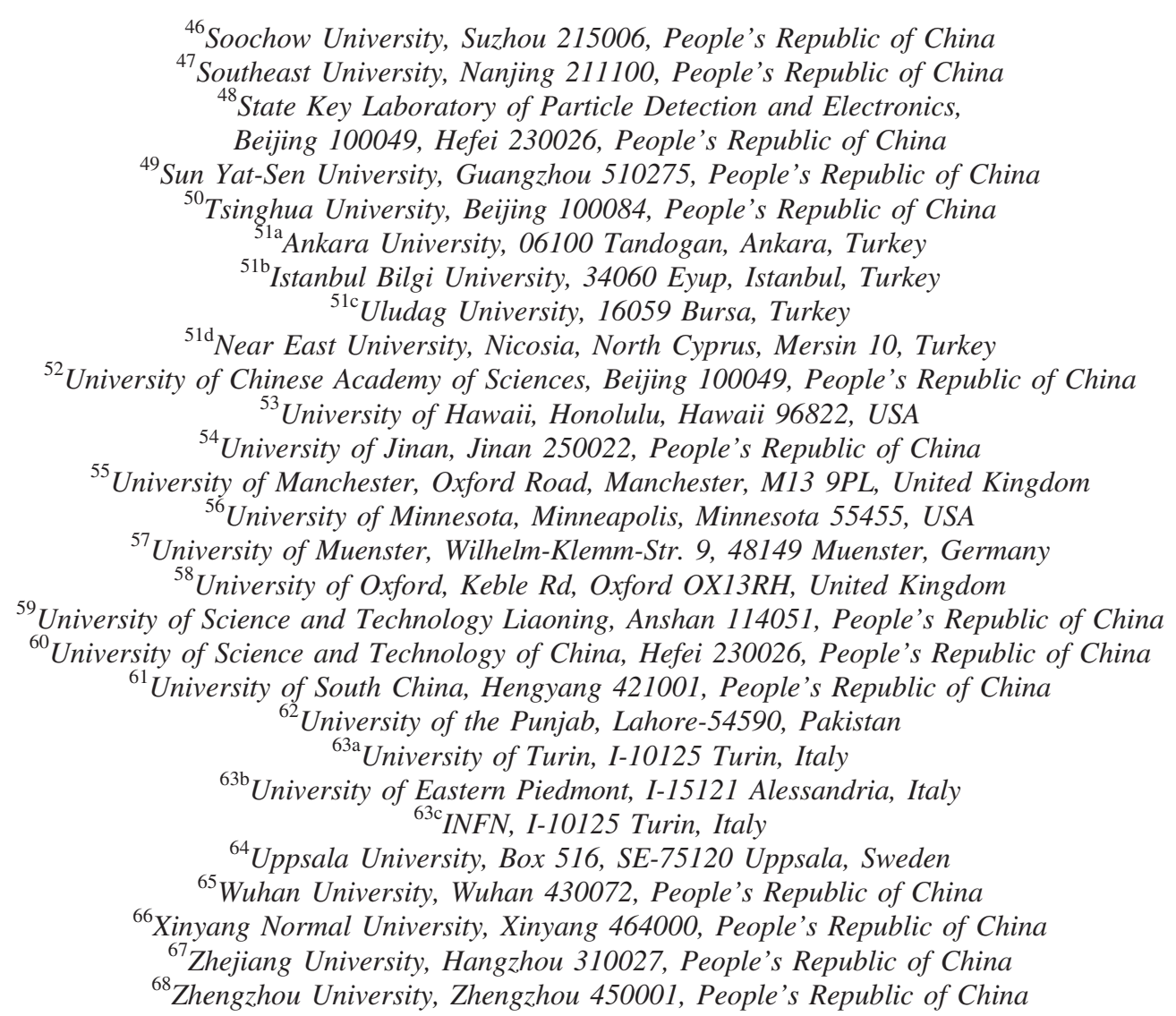

(Received 13 August 2020; accepted 3 November 2020; published 4 December 2020)

Using $2.93 \mathrm{fb}^{-1}$ of $e^{+} e^{-}$annihilation data collected at a center-of-mass energy $\sqrt{s}=3.773 \mathrm{GeV}$ with the BESIII detector operating at the BEPCII collider, we search for the semileptonic $D^{0(+)}$ decays into a $b_{1}(1235)^{-(0)}$ axial-vector meson for the first time. No significant signal is observed for either charge combination. The upper limits on the product branching fractions are $\mathcal{B}_{D^{0} \rightarrow b_{1}(1235)^{-} e^{+} \nu_{e}} \cdot \mathcal{B}_{b_{1}(1235)^{-} \rightarrow \omega \pi^{-}}<$ $1.12 \times 10^{-4}$ and $\mathcal{B}_{D^{+} \rightarrow b_{1}}(1235)^{0} e^{+} \nu_{e} \cdot \mathcal{B}_{b_{1}(1235)^{0} \rightarrow \omega \pi^{0}}<1.75 \times 10^{-4}$ at the $90 \%$ confidence level.

DOI: $10.1103 /$ PhysRevD.102.112005

${ }^{\mathrm{a}}$ Also at Bogazici University, 34342 Istanbul, Turkey.

${ }^{\mathrm{b}}$ Also at the Moscow Institute of Physics and Technology, Moscow 141700, Russia.

${ }^{\mathrm{c}}$ Also at the Novosibirsk State University, Novosibirsk 630090, Russia.

dAlso at the NRC "Kurchatov Institute", PNPI, 188300 Gatchina, Russia.

${ }^{\mathrm{e}}$ Also at Istanbul Arel University, 34295 Istanbul, Turkey.

${ }^{\mathrm{f}}$ Also at Goethe University Frankfurt, 60323 Frankfurt am Main, Germany.

${ }^{\mathrm{g}}$ Also at Key Laboratory for Particle Physics, Astrophysics and Cosmology, Ministry of Education; Shanghai Key Laboratory for Particle Physics and Cosmology; Institute of Nuclear and Particle Physics, Shanghai 200240, People's Republic of China.

${ }^{\mathrm{h}}$ Also at Key Laboratory of Nuclear Physics and Ion-beam Application (MOE) and Institute of Modern Physics, Fudan University, Shanghai 200443, People's Republic of China.

${ }^{\mathrm{i}}$ Also at Harvard University, Department of Physics, Cambridge, Massachusetts 02138, USA.

${ }^{j}$ Present address: Institute of Physics and Technology, Peace Ave. 54B, Ulaanbaatar 13330, Mongolia.

${ }^{k}$ Also at State Key Laboratory of Nuclear Physics and Technology, Peking University, Beijing 100871, People's Republic of China.

${ }^{1}$ School of Physics and Electronics, Hunan University, Changsha 410082, China.

Published by the American Physical Society under the terms of the Creative Commons Attribution 4.0 International license. Further distribution of this work must maintain attribution to the author(s) and the published article's title, journal citation, and DOI. Funded by SCOAP. 


\section{INTRODUCTION}

Semileptonic decays of the $D^{0(+)}$ provide an outstanding platform to explore the dynamics of both weak and strong interactions in the charm sector. The semileptonic $D^{0(+)}$ decays into pseudoscalar and vector mesons have been widely studied in both experiment [1] and theory. Extensive studies of the semileptonic $D^{0(+)}$ decays into axial-vector mesons $\bar{K}_{1}(1270)$ and $b_{1}(1235)$ play an important role in the understanding of nonperturbative strong-interaction dynamics in weak decays [2-8]. Nevertheless, knowledge of these decays is limited. The observation of the Cabibbofavored decay $D^{+} \rightarrow \bar{K}_{1}(1270)^{0} e^{+} \nu_{e}$ has been reported by the BESIII experiment [9], and evidence for $D^{0} \rightarrow$ $K_{1}(1270)^{-} e^{+} \nu_{e}$ has been found at CLEO [10]. The measured branching fractions are consistent with theoretical predictions based on the Isgur-Scora-Grinstein-Wise (ISGW) quark model [2] and its upgrade (ISGW2) [3], as well as those based on the covariant light-front quark model [6]. As for the singly Cabibbo-suppressed decays $D^{0(+)} \rightarrow b_{1}(1235)^{-(0)} e^{+} \nu_{e}$, no experimental study has yet been carried out. Experimental measurements of the semileptonic decays $D^{0(+)} \rightarrow b_{1}(1235)^{-(0)} e^{+} \nu_{e}$ are important to test theoretical calculations and to understand nonperturbative effects in heavy meson decays $[2,3,6]$.

In this paper, we report the first search for the semileptonic decays $D^{0} \rightarrow b_{1}(1235)^{-} e^{+} \nu_{e}$ and $D^{+} \rightarrow$ $b_{1}(1235)^{0} e^{+} \nu_{e}$. The data used in this analysis, corresponding to an integrated luminosity of $2.93 \mathrm{fb}^{-1}$ [11], was accumulated at a center-of-mass energy of $3.773 \mathrm{GeV}$ with the BESIII detector. Throughout this paper, charge conjugate channels are always implied.

\section{BESIII DETECTOR AND MONTE CARLO SIMULATION}

The BESIII detector is a magnetic spectrometer [12] located at the Beijing Electron Positron Collider (BEPCII) [13]. The cylindrical core of the BESIII detector consists of a helium-based multilayer drift chamber (MDC), a plastic scintillator time-of-flight system (TOF), and a CsI(Tl) electromagnetic calorimeter (EMC), which are all enclosed in a superconducting solenoidal magnet providing a $1.0 \mathrm{~T}$ magnetic field. The solenoid is supported by an octagonal flux-return yoke with resistive plate counter muon identifier modules interleaved with steel. The acceptance of charged particles and photons is $93 \%$ over $4 \pi$ solid angle. The charged-particle momentum resolution at $1 \mathrm{GeV} / c$ is $0.5 \%$, and the $d E / d x$ resolution is $6 \%$ for the electrons from Bhabha scattering. The EMC measures photon energies with a resolution of $2.5 \%(5 \%)$ at $1 \mathrm{GeV}$ in the barrel (end cap) region. The time resolution of the TOF barrel part is $68 \mathrm{ps}$, while that of the end cap part is $110 \mathrm{ps}$.

Simulated samples produced with the GEANT4-based [14] Monte Carlo (MC) package, which includes the geometric description of the BESIII detector and the detector response, are used to determine the detection efficiency and to estimate the backgrounds. The simulation includes the beam energy spread and initial state radiation (ISR) in the $e^{+} e^{-}$annihilations modeled with the generator KKMC [15]. The inclusive MC samples consist of the production of $D \bar{D}$ pairs with consideration of quantum coherence for all neutral $D$ modes, the non- $D \bar{D}$ decays of the $\psi(3770)$, the ISR production of the $J / \psi$ and $\psi(3686)$ states, and the continuum processes. The known decay modes are modeled with EvtGen [16] using the branching fractions taken from the Particle Data Group [1], and the remaining unknown decays from the charmonium states with LundCharm [17]. The final state radiations from charged final state particles are incorporated with the Рнотоs package [18]. The signal process $D^{0(+)} \rightarrow$ $b_{1}(1235)^{-(0)} e^{+} \nu_{e}$ is simulated with $b_{1}(1235)^{-(0)}$ decaying into $\omega \pi^{-(0)}$, using the ISGW2 model [3]. A relativistic Breit-Wigner function is used to parametrize the resonance $b_{1}(1235)^{-(0)}$, the mass and width of which are fixed to the world-average values of $1229.5 \pm 3.2 \mathrm{MeV} / c^{2}$ and $142 \pm 9 \mathrm{MeV}$, respectively [1].

\section{DATA ANALYSIS}

The process $e^{+} e^{-} \rightarrow \psi(3770) \rightarrow D \bar{D}$ provides an ideal opportunity to study semileptonic $D^{0(+)}$ decays with the double-tag (DT) method, because there are no additional particles that accompany the $D$ mesons in the final states [19]. Throughout the paper, $D$ denotes $D^{0}$ or $D^{+}$. At first, single-tag (ST) $\bar{D}^{0}$ mesons are reconstructed by using the hadronic decay modes of $\bar{D}^{0} \rightarrow K^{+} \pi^{-}, K^{+} \pi^{-} \pi^{0}$, and $K^{+} \pi^{-} \pi^{-} \pi^{+}$; while ST $D^{-}$mesons are reconstructed via the decays $D^{-} \rightarrow K^{+} \pi^{-} \pi^{-}, K_{S}^{0} \pi^{-}, K^{+} \pi^{-} \pi^{-} \pi^{0}, K_{S}^{0} \pi^{-} \pi^{0}$, $K_{S}^{0} \pi^{+} \pi^{-} \pi^{-}$, and $K^{+} K^{-} \pi^{-}$. Then the semileptonic $D$ candidates are reconstructed with the remaining tracks and showers. The candidate event in which $D$ decays into $b_{1}(1235) e^{+} \nu_{e}$ and $\bar{D}$ decays into a tag mode is called a DT event. Since the branching fraction of the subsequent decay $b_{1}(1235) \rightarrow \omega \pi$ is not well measured, the product of the branching fractions of the decay $D \rightarrow b_{1}(1235) e^{+} \nu_{e}\left(\mathcal{B}_{\mathrm{SL}}\right)$ and its subsequent decay $b_{1}(1235) \rightarrow \omega \pi\left(\mathcal{B}_{b_{1}}\right)$ is determined using

$$
\mathcal{B}_{\mathrm{SL}} \cdot \mathcal{B}_{b_{1}}=\frac{N_{\mathrm{DT}}}{N_{\mathrm{ST}}^{\mathrm{tot}} \cdot \bar{\varepsilon}_{\mathrm{SL}} \cdot \mathcal{B}_{\omega} \cdot\left(\mathcal{B}_{\pi^{0}}\right)^{k}},
$$

where $N_{\mathrm{ST}}^{\text {tot }}$ and $N_{\mathrm{DT}}$ are the yields of the ST $\bar{D}$ mesons and the DT signal events in data, respectively; $\mathcal{B}_{\omega}$ and $\mathcal{B}_{\pi^{0}}$ are the branching fractions of $\omega \rightarrow \pi^{+} \pi^{-} \pi^{0}$ and $\pi^{0} \rightarrow \gamma \gamma$, respectively; $k$ is the component, which corresponds to the number of $\pi^{0}$ mesons in the final states and $\bar{\varepsilon}_{\mathrm{SL}}$ is the average efficiency of reconstructing $D \rightarrow b_{1}(1235) e^{+} \nu_{e}$. The average signal efficiency, weighted over the tag modes $i$, is calculated by $\bar{\varepsilon}_{\mathrm{SL}}=\Sigma_{i}\left[\left(\varepsilon_{\mathrm{DT}}^{i} \cdot N_{\mathrm{ST}}^{i}\right) /\left(\varepsilon_{\mathrm{ST}}^{i} \cdot N_{\mathrm{ST}}^{\text {tot }}\right)\right]$, where 
$N_{\mathrm{ST}}^{i}$ is the ST yield of $\bar{D} \rightarrow i, \varepsilon_{\mathrm{ST}}^{i}$ is the detection efficiency of reconstructing $\bar{D} \rightarrow i$, and $\varepsilon_{\mathrm{DT}}^{i}$ is the detection efficiency of reconstructing $\bar{D} \rightarrow i$ and $D \rightarrow b_{1}(1235) e^{+} \nu_{e}$ at the same time.

The ST $\bar{D}$ candidates are selected with the same criteria employed in our previous works [9,20-28]. For each charged track (except for those used for reconstructing $K_{S}^{0}$ meson decays), the polar angle with respect to the MDC axis $(\theta)$ is required to satisfy $|\cos \theta|<0.93$, and the point of closest approach to the interaction point (IP) must be within $1 \mathrm{~cm}$ in the plan perpendicular to the MDC axis and within $\pm 10 \mathrm{~cm}$ along the MDC axis. Charged tracks are identified by using the $d E / d x$ and TOF information, with which the combined confidence levels under the pion and kaon hypotheses are computed separately. A charged track is assigned as the particle type which has a larger probability.

Candidate $K_{S}^{0}$ mesons are formed from pairs of oppositely charged tracks. For these two tracks, the distance of closest approach to the IP is required to be less than $20 \mathrm{~cm}$ along the MDC axis. No requirements on the distance of closest approach in the transverse plane or on particle identification (PID) criteria are applied to these tracks. The two charged tracks are constrained to originate from a common vertex, which is required to be away from the IP by a flight distance of at least twice the vertex resolution. The invariant mass of the $\pi^{+} \pi^{-}$pair is required to be within $(0.486,0.510) \mathrm{GeV} / c^{2}$.

Neutral pion candidates are reconstructed via the $\pi^{0} \rightarrow \gamma \gamma$ decays. Photon candidates are chosen from the EMC showers. The EMC time deviation from the event start time is required to be within $[0,700]$ ns. The energy deposited in the EMC is required to be greater than 25 (50) $\mathrm{MeV}$ if the crystal with the maximum deposited energy in that cluster is in the barrel (end cap) region [29]. The opening angle between the photon candidate and the nearest charged track is required to be greater than $10^{\circ}$. For any $\pi^{0}$ candidate, the invariant mass of the photon pair is required to be within $(0.115,0.150) \mathrm{GeV} / c^{2}$. To improve the momentum resolution, a mass-constrained (1-C) fit to the nominal $\pi^{0}$ mass [1] is imposed on the photon pair. The four-momentum of the $\pi^{0}$ candidate returned by this kinematic fit is used for further analysis.

In the selection of $\bar{D}^{0} \rightarrow K^{+} \pi^{-}$events, the backgrounds from cosmic rays and Bhabha events are rejected by using the same requirements described in Ref. [30]. To separate the ST $\bar{D}$ mesons from combinatorial backgrounds, we define the energy difference $\Delta E \equiv E_{\bar{D}}-E_{\text {beam }}$ and the beam-constrained mass $M_{\mathrm{BC}} \equiv \sqrt{E_{\text {beam }}^{2} / c^{4}-\left|\vec{p}_{\bar{D}}\right|^{2} / c^{2}}$, where $E_{\text {beam }}$ is the beam energy, and $E_{\bar{D}}$ and $\vec{p}_{\bar{D}}$ are the total energy and momentum of the ST $\bar{D}$ meson in the $e^{+} e^{-}$ center-of-mass frame. If there is more than one $\bar{D}$ candidate in a specific ST mode, the one with the least $|\Delta E|$ is kept for further analysis.
To suppress combinatorial backgrounds, the ST $\bar{D}$ candidates, which are reconstructed by using the modes with and without $\pi^{0}$ in the final states, are imposed with the requirements of $\Delta E \in(-0.055,0.045) \mathrm{GeV}$ and $\Delta E \in(-0.025,0.025) \mathrm{GeV}$, respectively. For each ST mode, the yield of ST $\bar{D}$ mesons is extracted by fitting the corresponding $M_{\mathrm{BC}}$ distribution. The signal is described by an MC-simulated shape convolved with a doubleGaussian function which compensates the resolution difference between data and MC simulation. The background is parametrized by the ARGUS function [31]. All fit parameters are left free in the fits. Figure 1 shows the fits to the $M_{\mathrm{BC}}$ distributions for individual ST modes. The candidates with $M_{\mathrm{BC}}$ lying in $(1.859,1.873) \mathrm{GeV} / c^{2}$ for $\bar{D}^{0}$ tags and $(1.863,1.877) \mathrm{GeV} / c^{2}$ for $D^{-}$tags are kept for further analysis. Summing over the tag modes, the total yields of ST $\bar{D}^{0}$ and $D^{-}$mesons are obtained to be $2321009 \pm$ $1875_{\text {stat }}$ and $1522474 \pm 2215_{\text {stat }}$, respectively [22].

We require that there are four and three charged tracks reconstructed in $D^{0} \rightarrow b_{1}(1235)^{-} e^{+} \nu_{e}$ and $D^{+} \rightarrow$ $b_{1}(1235)^{0} e^{+} \nu_{e}$ candidates, respectively. These tracks exclude those used to form the ST $\bar{D}$ candidates. For each candidate, one charged track is identified as a positron and the others are required to be identified as pions. The selection criteria of charged and neutral pions are the same as those used in selecting the ST $\bar{D}$ candidates. To suppress fake $\pi^{0}$ candidates, the decay angle of $\pi^{0}$, defined as

$$
\cos \theta_{\pi^{0}}=\left|E_{\gamma 1}-E_{\gamma 2}\right| /\left|\vec{p}_{\pi^{0}} \cdot c\right|,
$$

is required to be less than 0.9 . The requirement has been optimized using the inclusive MC sample. $E_{\gamma 1}$ and $E_{\gamma 2}$ are the energies of the two daughter photons of the $\pi^{0}$, and $\vec{p}_{\pi^{0}}$ is the reconstructed momentum of the $\pi^{0}$. For the selected
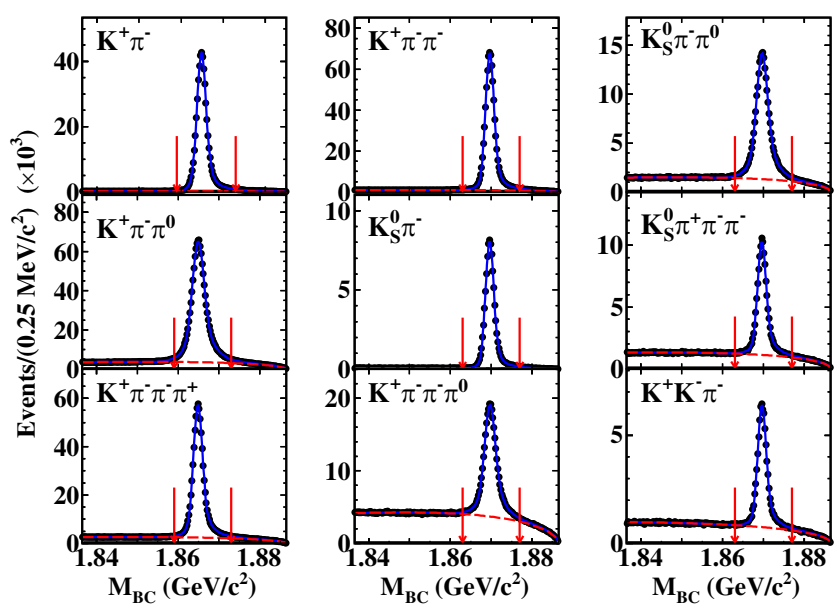

FIG. 1. Fits to the $M_{\mathrm{BC}}$ distributions of the ST $\bar{D}$ candidates. In each plot, the points with error bars are data, the red dashed curve is the background contribution, and the blue solid line shows the total fit. Pairs of red arrows show the $M_{\mathrm{BC}}$ signal windows. 

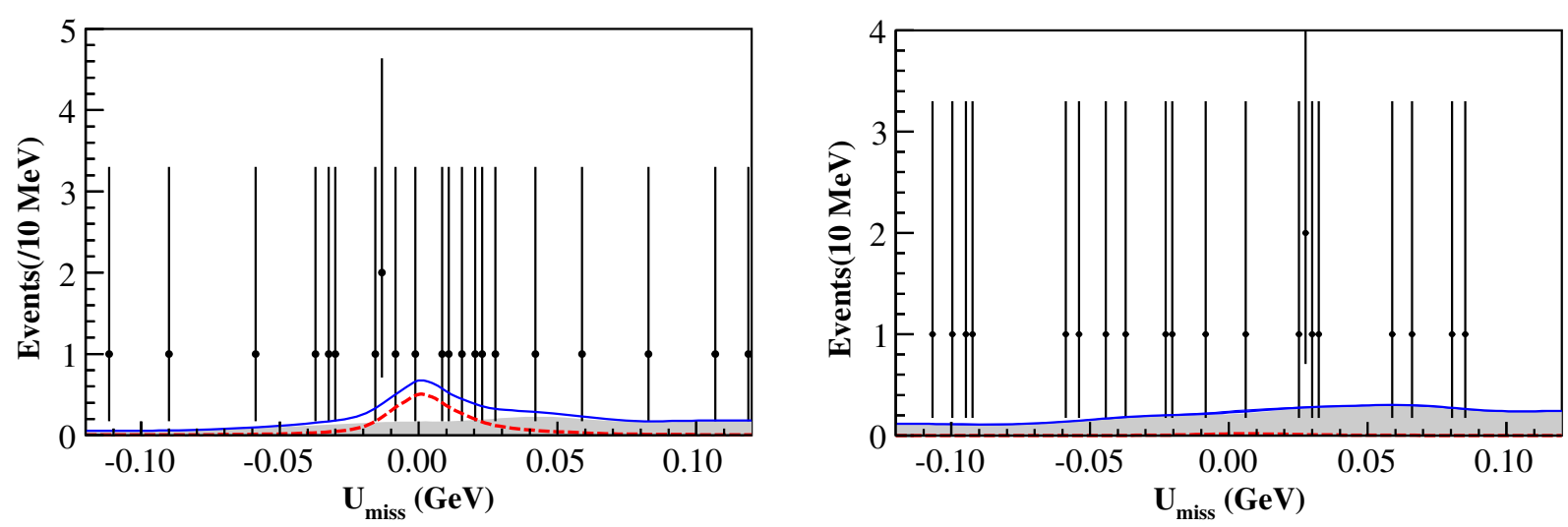

FIG. 2. Fits to the $U_{\text {miss }}$ distributions of the (left) $D^{0} \rightarrow b_{1}(1235)^{-} e^{+} \nu_{e}$ and (right) $D^{+} \rightarrow b_{1}(1235)^{0} e^{+} \nu_{e}$ candidate events. The points with error bars are data, the red dashed curve is the signal, the gray filled histogram is the background contribution, and the blue solid curve shows the total fit.

$D^{0} \rightarrow \pi^{+} \pi^{-} \pi^{-} \pi^{0} e^{+} \nu_{e}$ and $D^{+} \rightarrow \pi^{+} \pi^{-} \pi^{0} \pi^{0} e^{+} \nu_{e}$ candidates, there are always two possible $\pi^{+} \pi^{-} \pi^{0}$ combinations to form the $\omega$. The invariant masses of both combinations are required to be greater than $0.6 \mathrm{GeV} / c^{2}$ to suppress the backgrounds from $D \rightarrow a_{0}(980) e^{+} \nu_{e}$. One candidate is kept for further analysis if either of the combinations has an invariant mass falling in the $\omega$ mass signal region of $(0.757,0.807) \mathrm{GeV} / c^{2}$. To form a $b_{1}(1235)$ candidate, the $\omega \pi$ invariant mass is required to be within $(1.080,1.380) \mathrm{GeV} / c^{2}$. The background from $D^{0(+)} \rightarrow$ $\bar{K}_{1}(1270)\left[K_{S}^{0} \pi^{+(0)} \pi^{-(0)}\right] e^{+} \nu_{e}$ is rejected by requiring the invariant masses of any $\pi^{+} \pi^{-}\left(\pi^{0} \pi^{0}\right)$ combinations to be outside $(0.486,0.510) \mathrm{GeV} / c^{2}\left[(0.460,0.510) \mathrm{GeV} / c^{2}\right]$. These requirements correspond to three times the invariant mass resolution about the nominal $K_{\mathrm{S}}^{0}$ mass [1].

The $e^{+}$candidate is required to have a charge of opposite sign to that of the charm quark in the ST $\bar{D}$ meson. The $e^{+}$ candidate is identified by using the combined $d E / d x$, TOF, and EMC information. The combined confidence levels for the positron, pion, and kaon hypotheses $\left(C L_{e}, C L_{\pi}\right.$, and $\left.C L_{K}\right)$ are computed. The positron candidate is required to satisfy $C L_{e}>0.001$ and $C L_{e} /\left(C L_{e}+C L_{\pi}+C L_{K}\right)>0.8$. Its deposited energy in the $\mathrm{EMC}$ is required to be greater than 0.8 times its momentum reconstructed by the MDC, to further suppress the background from misidentified hadrons and muons.

The peaking backgrounds from hadronic $D$ decays with multiple pions in the final states are rejected by requiring that the invariant mass of $b_{1}(1235) e^{+}\left(M_{b_{1} e^{+}}\right)$is less than $1.80 \mathrm{GeV} / c^{2}$. To suppress backgrounds with extra photon(s), we require that the energy of any extra photon $\left(E_{\text {extra }}^{\gamma}\right)$ is less than $0.30 \mathrm{GeV}$ and there is no extra $\pi^{0}\left(N_{\text {extra }}^{\pi^{0}}\right)$ in the candidate event.

The neutrino is not detectable in the BESIII detector. To distinguish semileptonic signal events from backgrounds, we define $U_{\text {miss }} \equiv E_{\text {miss }}-\left|\vec{p}_{\text {miss }}\right| \cdot c$, where $E_{\text {miss }}$ and $\vec{p}_{\text {miss }}$ are the missing energy and momentum of the DT event in the $e^{+} e^{-}$center-of-mass frame, respectively. They are calculated as $E_{\text {miss }} \equiv E_{\text {beam }}-E_{b_{1}}-E_{e^{+}}$and $\quad \vec{p}_{\text {miss }} \equiv$ $\vec{p}_{D}-\vec{p}_{b_{1}}-\vec{p}_{e^{+}}$, where $E_{b_{1}\left(e^{+}\right)}$and $\vec{p}_{b_{1}\left(e^{+}\right)}$are the measured energy and momentum of the $b_{1}(1235)\left(e^{+}\right)$candidates, respectively, and $\vec{p}_{D} \equiv-\hat{p}_{\bar{D}} \cdot \sqrt{E_{\text {beam }}^{2} / c^{2}-m_{\bar{D}}^{2} \cdot c^{2}}$, where $\hat{p}_{\bar{D}}$ is the unit vector in the momentum direction of the ST $\bar{D}$ meson and $m_{\bar{D}}$ is the nominal $\bar{D}$ mass [1]. The use of the beam energy and the nominal $D$ mass for the magnitude of the ST $D$ mesons improves the $U_{\text {miss }}$ resolution. For the correctly reconstructed signal events, $U_{\text {miss }}$ peaks at zero.

Figure 2 shows the $U_{\text {miss }}$ distributions of the accepted candidate events. Unbinned maximum likelihood fits are performed on these distributions. In the fits, the signal and background are modeled by the simulated shapes obtained from the signal MC events and the inclusive MC sample, respectively, and the yields of the signal and background are left free. Since no significant signal is observed, conservative upper limits will be set by assuming all the fitted signals are from $b_{1}(1235)$.

The detection efficiencies $\bar{\varepsilon}_{\mathrm{SL}}$ are estimated to be $0.0704 \pm 0.0006$ and $0.0412 \pm 0.0002$ for the $D^{0} \rightarrow$ $b_{1}(1235)^{-} e^{+} \nu_{e}$ and $D^{+} \rightarrow b_{1}(1235)^{0} e^{+} \nu_{e}$ decays, respectively. The blue dotted curves in Fig. 3 show the raw likelihood distributions versus the corresponding product of branching fractions.

\section{SYSTEMATIC UNCERTAINTY}

With the DT method, many systematic uncertainties on the ST side mostly cancel. The sources of the systematic uncertainties in the measurements of the product of branching fractions are classified into two cases. The first one is from the uncertainties relying on effective efficiencies and are assigned relative to the measured branching fractions. The uncertainty associated with the ST yield $N_{\text {ST }}^{\text {tot }}$ is estimated to be $0.5 \%$ [20-22]. The uncertainty from the quoted branching fraction of the $\omega \rightarrow \pi^{+} \pi^{-} \pi^{0}$ decay is 

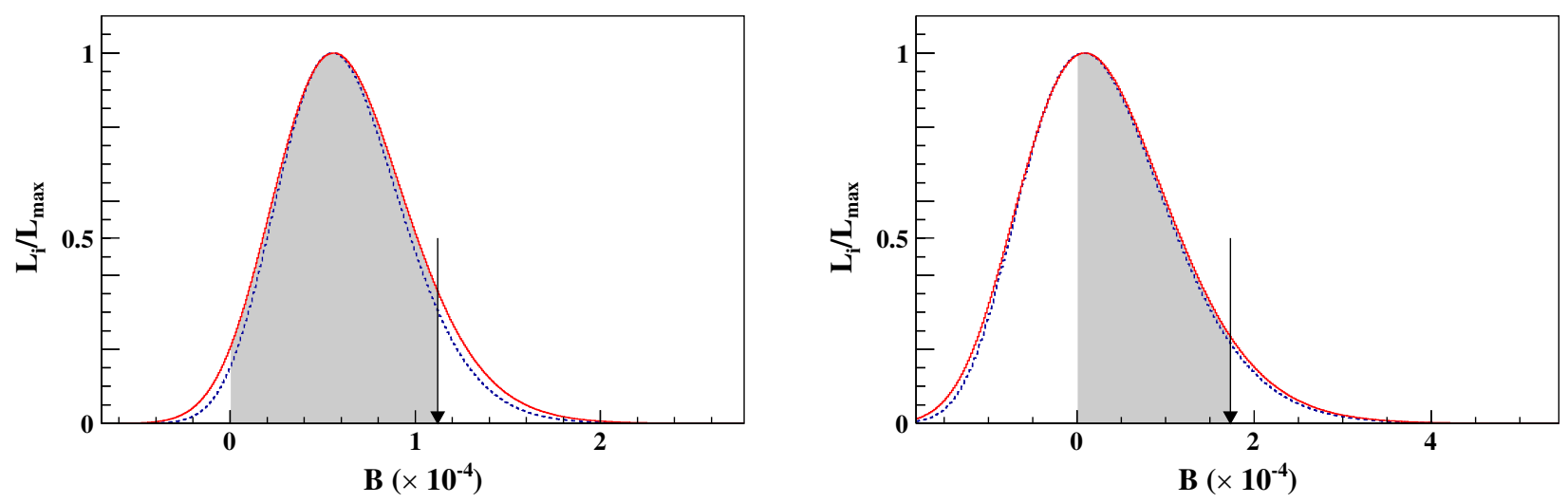

FIG. 3. Likelihood distributions versus the corresponding product of branching fractions for (left) $D^{0} \rightarrow b_{1}(1235)^{-} e^{+} \nu_{e}$ and (right) $D^{+} \rightarrow b_{1}(1235)^{0} e^{+} \nu_{e}$, with (red solid curves) and without (blue dotted curves) smearing the systematic uncertainties. The black arrows correspond to the upper limits at the $90 \%$ confidence level.

$0.8 \%$. The uncertainties from the tracking and PID of $e^{ \pm}$ are studied with a control sample of $e^{+} e^{-} \rightarrow \gamma e^{+} e^{-}$. The uncertainties from the tracking and PID of $\pi^{ \pm}$and $\pi^{0}$ reconstruction are obtained by studying a DT control sample $\psi(3770) \rightarrow D \bar{D}$ with hadronic $D$ decays $[20,21]$. The systematic uncertainties from the tracking (PID) efficiencies are assigned as $1.0 \%(1.0 \%)$ per $e^{ \pm}$and $1.0 \%(1.0 \%)$ per $\pi^{ \pm}$, respectively. The $\pi^{0}$ reconstruction efficiencies include photon finding, the $\pi^{0}$ mass window, and the 1-C kinematic fit, the systematic uncertainty of which is taken to be $2.0 \%$ per $\pi^{0}$. The systematic uncertainty from the $\pi^{0}$ decay angle requirement is determined to be $2.0 \%$ per $\pi^{0}$ by studying the DT events of $D^{0} \rightarrow$ $K^{-} \pi^{+} \pi^{0}$ versus $\bar{D}^{0} \rightarrow K^{+} \pi^{-}$and $K^{+} \pi^{-} \pi^{-} \pi^{+}$. The systematic uncertainty associated with the $\omega$ mass window is assigned to be $1.2 \%$ using a control sample of $D^{0} \rightarrow K_{S}^{0} \omega$ reconstructed versus the same $\bar{D}^{0}$ tags as those used in the nominal analysis. The systematic uncertainties from the $E_{\text {extra } \gamma}^{\max }$ and $N_{\text {extra, } \pi^{0}}$ requirements are estimated to be $1.4 \%$ and $2.0 \%$ for $D^{0} \rightarrow b_{1}(1235)^{-} e^{+} \nu_{e}$ and $D^{+} \rightarrow$ $b_{1}(1235)^{0} e^{+} \nu_{e}$, respectively, which are estimated using DT samples of $D^{0} \rightarrow K^{-} e^{+} \nu_{e}$ and $D^{+} \rightarrow K_{S}^{0} e^{+} \nu_{e}$ decays reconstructed versus the same tags as the nominal analysis. The systematic uncertainty related to the MC generator is estimated using alternative signal MC samples, which are produced by varying the mass and width of the $b_{1}(1235)$ by $\pm 1 \sigma$. The maximum changes of the signal efficiencies, $5.1 \%$ and $2.7 \%$, are assigned as the systematic uncertainties for $D^{0} \rightarrow b_{1}(1235)^{-} e^{+} \nu_{e}$ and $D^{+} \rightarrow b_{1}(1235)^{0} e^{+} \nu_{e}$, respectively. The uncertainties from limited MC statistics, propagated from those of the ST and DT efficiencies, are $0.7 \%$ and $0.9 \%$ for $D^{0} \rightarrow b_{1}(1235)^{-} e^{+} \nu_{e}$ and $D^{+} \rightarrow$ $b_{1}(1235)^{0} e^{+} \nu_{e}$, respectively. By adding these uncertainties in quadrature, the total systematic errors associated with the signal efficiencies $\left(\sigma_{\epsilon}\right)$ are obtained to be $8.2 \%$ and $7.3 \%$ for $D^{0} \rightarrow b_{1}(1235)^{-} e^{+} \nu_{e}$ and $D^{+} \rightarrow b_{1}(1235)^{0} e^{+} \nu_{e}$, respectively.
The second kind of systematic uncertainty originates from the fit to the $U_{\text {miss }}$ distribution of the semileptonic $D$ decay candidates. It is dominated by the uncertainty from imperfect knowledge of the background shape. The uncertainty associated with the signal shape is negligible. The background shape is obtained from the inclusive MC sample using a kernel estimation method [32] implemented in RooFit [33]. Unlike the other sources of uncertainties, the background shape directly affects the likelihood function. The smoothing parameter of RooKeysPdf is varied within a reasonable range to obtain alternative background shapes. The absolute change of the signal yield, which gives the largest upper limit on the branching fraction, is taken as the systematic uncertainty $\left(\sigma_{n}\right)$. It is found to be 1.7 for $D^{0} \rightarrow b_{1}(1235)^{-} e^{+} \nu_{e}$ and 1.1 for $D^{+} \rightarrow b_{1}(1235)^{0} e^{+} \nu_{e}$.

\section{RESULTS}

To take into account the first kind of systematic uncertainty in the calculation of the upper limits, the raw likelihood distribution versus the product of branching fractions is smeared by a Gaussian function with a mean of 0 and a width equal to $\sigma_{\epsilon}$ according to Refs. [34,35].

To incorporate the second kind of systematic uncertainty, the updated likelihood is then convolved with another Gaussian function with mean of 0 and a width equal to $\sigma_{\mathcal{B}}$ similarly. Here $\sigma_{\mathcal{B}}$ is an uncertainty of the product of the branching fractions calculated with Eq. (1) by replacing $N_{\text {DT }}$ with $\sigma_{n}$.

The red solid curves in Fig. 3 show the resulting likelihood distributions for the two decays. The upper limits on the product of branching fractions at the $90 \%$ confidence level (C.L.), obtained by integrating $L(\mathcal{B})$ from zero to $90 \%$ of the total curve, are

$$
\mathcal{B}_{D^{0} \rightarrow b_{1}(1235)^{-} e^{+} \nu_{e}} \cdot \mathcal{B}_{b_{1}(1235)^{-} \rightarrow \omega \pi^{-}}<1.12 \times 10^{-4}
$$


and

$$
\mathcal{B}_{D^{+} \rightarrow b_{1}(1235)^{0} e^{+} \nu_{e}} \cdot \mathcal{B}_{b_{1}(1235)^{0} \rightarrow \omega \pi^{0}}<1.75 \times 10^{-4} .
$$

\section{SUMMARY}

In summary, by analyzing $2.93 \mathrm{fb}^{-1}$ of $e^{+} e^{-}$collision data taken at $\sqrt{s}=3.773 \mathrm{GeV}$ with the BESIII detector, the semileptonic $D^{0(+)}$ decays into axial-vector mesons $b_{1}(1235)^{-(0)}$ have been searched for the first time. Since no significant signal is observed, the upper limits on the product of branching fractions for $D^{0} \rightarrow b_{1}(1235)^{-} e^{+} \nu_{e}$ and $D^{+} \rightarrow b_{1}(1235)^{0} e^{+} \nu_{e}$ at the $90 \%$ C.L. are estimated to be $\mathcal{B}_{D^{0} \rightarrow b_{1}(1235)^{-} e^{+} \nu_{e}} \cdot \mathcal{B}_{b_{1}(1235)^{-} \rightarrow \omega \pi^{-}}<1.12 \times 10^{-4}$ and $\mathcal{B}_{D^{+} \rightarrow b_{1}(1235)^{0} e^{+} \nu_{e}} \cdot \mathcal{B}_{b_{1}(1235)^{0} \rightarrow \omega \pi^{0}}<1.75 \times 10^{-4}$, respectively. When assuming $\mathcal{B}_{b_{1}(1235) \rightarrow \omega \pi}=1$, these results are comparable with the theoretical prediction in Ref. [6]. It is anticipated that these decays could be observed with larger data samples at BESIII [36] and Belle II [37].

\section{ACKNOWLEDGMENTS}

The BESIII collaboration thanks the staff of BEPCII and the IHEP computing center for their strong support. This work is supported in part by National Key Basic Research Program of China under Contract No. 2020YFA0406300 and No 2020YFA0406400; National Natural Science Foundation of China (NSFC) under Contracts
No. 11805037, No. 11775230, No. 11625523, No. 11635010, No. 11735014, No. 11822506, No. 11835012, No. 11935015, No. 11935016, No. 11935018, No. 11961141012; the Chinese Academy of Sciences (CAS) Large-Scale Scientific Facility Program; Joint Large-Scale Scientific Facility Funds of the NSFC and CAS under Contracts No. U1832121, No. U1732263, No. U1832207; CAS Key Research Program of Frontier Sciences under Contracts No. QYZDJ-SSW-SLH003, No. QYZDJ-SSW-SLH040; 100 Talents Program of CAS; Open Research Program of Large Research Infrastructures, Chinese Academy of Sciences; Institute of Nuclear and Particle Physics at Shanghai Jiao Tong University (INPAC) and Shanghai Key Laboratory for Particle Physics and Cosmology; ERC under Contract No. 758462; German Research Foundation DFG under Contracts No. 443159800, Collaborative Research Center CRC 1044, FOR 2359, FOR 2359, GRK 214; Istituto Nazionale di Fisica Nucleare, Italy; Ministry of Development of Turkey under Contract No. DPT2006K120470; National Science and Technology fund; Olle Engkvist Foundation under Contract No. 200-0605; STFC (United Kingdom); The Knut and Alice Wallenberg Foundation (Sweden) under Contract No. 2016.0157; The Royal Society, UK under Contracts No. DH140054, No. DH160214; The Swedish Research Council; U.S. Department of Energy under Contracts No. DE-FG02-05ER41374, No. DE-SC-0012069.
[1] P. A. Zyla et al. (Particle Data Group), Prog. Theor. Exp. Phys. (2020), 083C01.

[2] N. Isgur, D. Scora, B. Grinstein, and M. B. Wise, Phys. Rev. D 39, 799 (1989).

[3] D. Scora and N. Isgur, Phys. Rev. D 52, 2783 (1995).

[4] R. Khosravi, K. Azizi, and N. Ghahramany, Phys. Rev. D 79, 036004 (2009).

[5] Y. B Zuo, Y. Hu, L. L. He, W. Yang, Y. Chen, and Y. N. Hao, Int. J. Mod. Phys. A 31, 1650116 (2016).

[6] H. Y. Cheng and X. W. Kang, Eur. Phys. J. C 77, 587 (2017); 77, 863(E) (2017).

[7] S. Momeni and R. Khosravi, J. Phys. G 46, 105006 (2019).

[8] S. Momeni, J. Phys. G 46, 105006 (2019).

[9] M. Ablikim et al. (BESIII Collaboration), Phys. Rev. Lett. 123, 231801 (2019).

[10] M. Artuso et al. (CLEO Collaboration), Phys. Rev. Lett. 99, 191801 (2007).

[11] M. Ablikim et al. (BESIII Collaboration), Chin. Phys. C 37, 123001 (2013); Phys. Lett. B 753, 629 (2016).

[12] M. Ablikim et al. (BESIII Collaboration), Nucl. Instrum. Methods Phys. Res., Sect. A 614, 345 (2010).

[13] C. H. Yu et al., Proceedings of IPAC2016, Busan, Korea (2016), https://doi.org/10.18429/JACoW-IPAC2016-TUYA01.
[14] S. Agostinelli et al. (GEANT4 Collaboration), Nucl. Instrum. Methods Phys. Res., Sect. A 506, 250 (2003).

[15] S. Jadach, B. F. L. Ward, and Z. Was, Comput. Phys. Commun. 130, 260 (2000); Phys. Rev. D 63, 113009 (2001).

[16] D. J. Lange, Nucl. Instrum. Methods Phys. Res., Sect. A 462, 152 (2001); R. G. Ping, Chin. Phys. C 32, 599 (2008).

[17] J. C. Chen, G. S. Huang, X. R. Qi, D. H. Zhang, and Y. S. Zhu, Phys. Rev. D 62, 034003 (2000).

[18] E. Richter-Was, Phys. Lett. B 303, 163 (1993).

[19] R. M. Baltrusaitis et al. (MARK-III Collaboration), Phys. Rev. Lett. 56, 2140 (1986); J. Adler et al. (MARK-III Collaboration), Phys. Rev. Lett. 60, 89 (1988).

[20] M. Ablikim et al. (BESIII Collaboration), Eur. Phys. J. C 76, 369 (2016).

[21] M. Ablikim et al. (BESIII Collaboration), Chin. Phys. C 40, 113001 (2016).

[22] M. Ablikim et al. (BESIII Collaboration), Phys. Rev. Lett. 121, 171803 (2018).

[23] M. Ablikim et al. (BESIII Collaboration), Phys. Rev. D 101, 052009 (2020).

[24] M. Ablikim et al. (BESIII Collaboration), Phys. Rev. D 101, 072005 (2020). 
[25] M. Ablikim et al. (BESIII Collaboration), Phys. Rev. Lett. 124, 231801 (2020).

[26] M. Ablikim et al. (BESIII Collaboration), Phys. Rev. Lett. 124, 241803 (2020).

[27] M. Ablikim et al. (BESIII Collaboration), Phys. Rev. Lett. 125, 141802 (2020).

[28] M. Ablikim et al. (BESIII Collaboration), Phys. Rev. D 102, 052006 (2020).

[29] M. Ablikim et al. (BESIII Collaboration), Nucl. Instrum. Methods Phys. Res., Sect. A 614, 345 (2010).

[30] M. Ablikim et al. (BESIII Collaboration), Phys. Lett. B 734, 227 (2014).
[31] H. Albrecht et al. (ARGUS Collaboration), Phys. Lett. B 241, 278 (1990).

[32] K. S. Cranmer, Comput. Phys. Commun. 136, 198 (2001).

[33] R. Brun and F. Rademakers, Nucl. Instrum. Methods Phys. Res., Sect. A 389, 81 (1997).

[34] K. Stenson, arXiv:physics/0605236.

[35] X. X. Liu, X. R. Lyu, and Y. S. Zhu, Chin. Phys. C 39, 103001 (2015).

[36] M. Ablikim et al. (BESIII Collaboration), Chin. Phys. C 44, 040001 (2020).

[37] E. Kou et al. (Belle II Collaboration), Prog. Theor. Exp. Phys. (2019), 123C01. 